Revue internationale de l'économie sociale

Recma

\title{
Habitat groupé et organismes HLM : une utopie réalisable et reproductible?
}

\section{Emilie Cariou}

Numéro 323, janvier 2012

URI : https://id.erudit.org/iderudit/1018341ar

DOI : https://doi.org/10.7202/1018341ar

Aller au sommaire du numéro

Éditeur(s)

Association Recma

ISSN

1626-1682 (imprimé)

2261-2599 (numérique)

Découvrir la revue

\section{Citer cet article}

Cariou, E. (2012). Habitat groupé et organismes HLM : une utopie réalisable et reproductible ? Revue internationale de l'économie sociale, (323), 29-33.

https://doi.org/10.7202/1018341ar 


\title{
HABITAT GROUPÉ ET ORGANISMES HLM: UNE UTOPIE RÉALISABLE ET REPRODUCTIBLE?
}

\author{
par Emilie Cariou*
}

*Etudiante, titulaire d'unmaster
2 mention administration, mana-
gement et économie des orga-
nisations, spécialité manage-
ment des entreprisesmutualistes
et coopératives, IAE de Bretagne-
Occidentale. Mél.:emilie_cariou@
yahoo.com.
Le travail présenté dans cette
communication a été réalisé dans
le cadre de l'Association pour le
développement de l'économie
sociale et solidaire.
(1) Afin de mener cette étude de
façon coopérative et participative,
un comité de pilotage a été mis
en place pour valider le travail
effectué, échanger et alimenter
les diverses réflexions. Parmi
les participants figurent des por-
teurs de projets d'habitat groupé,
des structures mettant en place
l'habitat groupé, la Cres Bretagne
antenne Finistère, une maître de
conférences en sociologie, deux
doctorantes, une personne inté-
ressée par l'habitat groupé et des
membres de l'Adess pays de Brest.

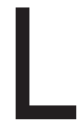
'Association pour le développement de l'économe sociale et solidaire (Adess) a pour mission de déterminer, d'initier et de mettre en œuvre des stratégies de coopération et de développement du réseau des acteurs de l'économie sociale et solidaire (ESS) du pays de Brest. Dans le cadre de ses missions, elle a souhaité travailler sur l'habitat groupé, qui, bien qu'expérimental en France, connaît un succès grandissant. Plus précisément, nous nous sommes engagés sur une étude de faisabilité juridique ${ }^{(1)}$ étudiant la relation complexe entre les porteurs de projets d'habitat groupé et les organismes HLM.

L'habitat groupé, tel que nous avons pu le définir, se désigne comme tout projet collectif de logements (en accession à la propriété ou en location, avec ou sans mise en place de logements conventionnés) dont les habitants s'impliquent dans la promotion et la gestion (mutualisation des finances, des espaces, des services, du temps...) pour créer un lieu de vie solidaire, écologique, adapté et pérenne ${ }^{(2)}$. Le meilleur moyen, pour les porteurs de projet, de parvenir à constituer ce lieu de vie est de travailler en collaboration avec des organismes HLM, dans la mesure où ceux-ci doivent répondre aux mêmes problématiques.

\section{Les difficultés rencontrées par les projets d'habitat groupé}

(2) Plusieurs vocables existent: certains parlent d'habitat coopératif, d'habitat solidaire, d'habitat groupé ou encore de coopérative d'habitants.
Nous avons recensé et inscrit dans une base de données 311 projets d'habitat groupé. Géographiquement, ces projets se situent majoritairement en Rhône-Alpes (63), en Bretagne (48) et en Ile-de-France (43). Quasiment toutes les régions sont représentées, à l'exception de la HauteNormandie et des cinq régions d'outre-mer. Parmi les départements où se situent le plus de projets se trouvent l'Isère, avec 23 projets, suivi de la Loire-Atlantique (20 projets), du Rhône et de l'Ille-et-Vilaine (18 projets). En France, $90 \%$ des projets d'habitat groupé sont initiés par les habitants, $8 \%$ le sont par les collectivités locales.

Sur les 184 questionnaires, nous avons obtenu 22 réponses concernant les réseaux de partenaires entretenus par les porteurs de projet. Tous 
(3) Parmi les groupes recensés, $10 \%(32)$ possèdent ainsi un double statut. II existe douze formules : SCIA-association, SCIA copropriété, SCCC-copropriété, $\mathrm{SCl}$-association, $\mathrm{SCl}$-copropriété, Scip-association, indivision-ASL, indivision-copropriété, SARL association, Sciapp-copropriété, Sciapp-association, convention organisme HLM-association. collaborent avec les collectivités locales. Une moitié des répondants ont eu, ou ont encore, des liens avec les organismes HLM. Les trois quarts sont en relation avec des structures accompagnatrices (Réseau Habitat groupé, Eco habitat groupé, Groupement des ressources techniques de l'Ouest, Habicoop...). La population est aussi sollicitée par treize groupes, pour de l'information ou de la communication autour de l'habitat groupé. D'autres partenaires existent, parmi lesquels des architectes, des associations comme Habitat et Humanisme ou l'Association des paralysés de France. Schématiquement, les porteurs de projet et leurs partenaires sont confrontés à plusieurs types de difficultés.

\section{Le millefeuille juridique}

Le droit français ne possédant pas de cadre juridique propre à l'habitat groupé, il faut bien souvent concilier deux statuts ${ }^{(3)}$, l'un pour l'habitation et l'autre pour la construction, ou encore un statut pour les espaces communs et un autre pour les parties privatives. Pour la moitié des groupes recensés (51\%), nous n'avons pas accès à cette information. Pour l'autre moitié, les statuts les plus usités se présentent ainsi : la société civile immobilière $(\mathrm{SCI}, 21 \mathrm{cas})$, la copropriété $(15 \mathrm{cas})$, l'association (13 cas), la société civile immobilière d'accession (SCIA, 9 cas). Viennent ensuite l'association syndicale libre (ASL, 4 cas), la société à responsabilité limitée (SARL), la société civile immobilière d'accession progressive à la propriété (Sciapp) et la société coopérative d'intérêt collectif (Scic, 3 cas), l'indivision et la société civile coopérative de construction (SCCC, 2 cas). Enfin, $17 \%$ des groupes opteraient pour le statut de coopérative d'habitants, s'il existait.

\section{La pérennité du groupe}

En principe, les partenaires d'un projet d'habitat classique sont confrontés à une ou deux personnes physiques ou morales. Or, dans ces projets d'habitat, c'est tout un collectif plus ou moins bien stabilisé et pérenne qui se présente à eux. Il arrive que cet ensemble soit fragilisé par des départs et des arrivées, et ce changement peut contribuer à la perte de partenaires. Lors de nos entretiens, certains organismes HLM nous ont confirmé que le changement fréquent d'interlocuteurs nuisait à la qualité de leurs relations avec les groupes.

\section{Concilier des intérêts multiples}

De nombreux porteurs de projet proposent en téléchargement un questionnaire individuel sur les attentes de la personne qui espère intégrer un habitat groupé. Ce questionnaire est le préalable à toute rencontre physique. La conception de ce formulaire se fait en deux temps, prenant d'abord en compte l'intérêt individuel de chaque membre du groupe - par le biais du questionnaire en téléchargement - et ensuite l'intérêt collectif. Une fois que ces deux intérêts ont été définis et ont reçu l'approbation de tous, il faut que ces demandes soient cohérentes vis-à-vis des 
autres partenaires. L'intérêt de tous sera donc la combinaison des intérêts personnels, de l'intérêt collectif et des intérêts des autres partenaires.

\section{La différence de temporalité}

D'un côté, il y a des professionnels et des techniciens et, de l'autre, des habitants ou futurs habitants. Une certaine méfiance réciproque peut, au départ, paralyser le projet. Cela nécessite donc beaucoup de communication, de pédagogie et de temps. Entre trois et cinq ans séparent le projet d'habitat groupé de sa réalisation.

\section{Le financement}

Quelle que soit la situation géographique des groupes, tous sont soumis à cette difficulté. Acheter un terrain ou un bâti existant est complexe, ainsi que financer les espaces communs ou faire face aux surcoûts de l'emploi de matériaux écologiques, d'autant que les aides et les subventions demeurent exceptionnelles.

\section{L'intégration de logements sociaux}

La mise en place de logements sociaux est souhaitée ou envisagée par $32 \%$ des groupes recensés, contre $34 \%$ qui ne la souhaitent pas. Les $34 \%$ restants correspondent aux groupes pour lesquels nous ne possédons pas l'information. L'attribution des logements sociaux est un des problèmes majeurs des groupes souhaitant intégrer des logements sociaux. Il convient de préciser que l'attribution est soumise à un comité réglementé par la loi et souverain dans sa décision.

Concrètement, pour les porteurs de projet d'habitat groupé, cela entraîne deux conséquences majeures: les membres porteurs de projet ne peuvent se coopter et les futurs habitants retenus par le comité d'attribution ne peuvent participer à la programmation. Cela étant, une fois les critères légaux et règlementaires respectés, les programmes d'accession peuvent prendre en compte d'autres éléments, comme la motivation au projet participatif ou l'engagement à long terme.

\section{Pourquoi établir des partenariats habitat groupé-HLM?}

(4) Devaux C., "Accompagner les projets d'habitat coopératif et participatif " FNSCHLM \& USH, février 2011.
L'organisme HLM et le groupe porteur de projet répondent véritablement aux enjeux actuels de la société: favoriser l'accession au logement locatif et à la propriété, renforcer la cohésion sociale, favoriser la mixité sociale et générationnelle, rendre accessibles des logements sains et économes au plus grand nombre ${ }^{(4)}$.

\section{Crédibiliser les projets, faciliter l'accès au foncier, assurer la mixité}

Pour les collectifs d'habitat groupé, insérer un organisme HLM dans leurs projets peut permettre de surmonter les difficultés évoquées. Il 
(5) Pour les groupes porteurs de projet qui ont ou qui envisagent un partenariat avec les organismes HLM, l'absence de modèle de convention est problématique. II n'existe pas de convention-type, néanmoins il est possible de trouver des modèles auprès de groupes qui travaillent en partenariat depuis plusieurs années. (6) Les groupes qui ne peuvent pas ou ne le souhaitent pas peuvent se constituer en bailleurs privés. peut s'agir tout d'abord de permettre aux ménages à bas revenus de participer à l'élaboration du programme architectural d'une autre forme d'habitat reposant sur la coopération, la mixité sociale, culturelle ou intergénérationnelle, la solidarité, notamment financière, et le respect d'un certain nombre de critères écologiques.

La présence des organismes HLM peut également apporter crédibilité et légitimité au projet, car l'habitat groupé est encore expérimental en France et les partenaires, surtout bancaires, peuvent se montrer frileux. Le partenariat peut également réduire les coûts relatifs au foncier. Les organismes HLM ont en effet des conditions particulières d'accès à celui-ci, permettant d'économiser sur les frais d'acquisition du terrain. En outre, concernant les dépenses relatives au logement social, ces opérateurs ont la capacité d'obtenir des financements de type PLAI (prêt locatif aidé d'intégration) ou PLUS (prêt locatif à usage social) que les personnes privées, mêmes regroupées en société, ne peuvent obtenir.

\section{Innovations et pérennité pour les organismes HLM}

Du point de vue des organismes HLM, s'intégrer dans des projets d'habitat groupé présente également un intérêt évident, en raison de l'implication des futurs habitants, qui participent à la conception des logements avant de les intégrer. Portés par une dynamique forte et un intérêt marqué pour la propriété collective plutôt qu'individuelle, ces projets apportent une plus-value remarquable au programme. Que ce soit en termes de durabilité, d'entretien ou d'habitation, ce sont des exemples d'habitats écologiques et économiques voués à la pérennisation de l'habitat. Ainsi, pour les opérations en location, il y a moins de mobilité qu'auparavant dans le logement social public. L'habitat groupé peut donc présenter une alternative pour améliorer le taux de rotation, pour les projets intégrant la location. Enfin, ce mode d'habitat permet aux organismes HLM souhaitant innover d'allier leurs aspirations économiques aux aspirations écologiques de l'habitat groupé. Certains parlent même de " projets-vitrines ». Ce scénario fonctionne actuellement ${ }^{(5)}$ dans le cadre d'opérations d'accession à la propriété. La plupart des groupes qui envisagent un partenariat avec un organisme HLM ${ }^{(6)}$ décident de se faire conseiller par un professionnel. Cet accompagnement peut s'effectuer du montage du groupe jusqu'à la livraison des logements, mais il peut également intervenir jusqu'à une année après celle-ci, afin d'apprendre au groupe la vie en collectif, les méthodes d'animation, la gestion de conflits... L'aide d'un professionnel semble un élément très favorable de réussite pour le projet.

\section{Conclusion}

En dépit du souhait de nombreux groupes porteurs de projet, aucun programme d'habitat groupé n'est réellement reproductible à l'identique. La complexité juridique des opérations l'explique en partie, comme le fait 
que, pour de nombreux organismes HLM, l'habitat groupé reste une alternative anecdotique et qu'il n'y a pas lieu, pour eux, de développer un partenariat avec ces groupes. Sur tous les groupes recensés qui ont travaillé avec un organisme HLM, seulement un ou deux des programmes en location conservent de bonnes relations avec lui.

Lexistence d'un statut juridique propre à l'habitat groupé, comme la coopérative d'habitants, pourrait faciliter la reproductibilité des projets, de même que l'existence d'une pratique foncière spécifique. Ce système, qui existe déjà dans le domaine agricole, permet l'installation d'agriculteurs via une part d'investissement éthique et solidaire. En leur absence, la société coopérative d'intérêt collectif (Scic) nous apparaît comme la forme la plus à même de concilier les intérêts multiples qui s'expriment à travers ces projets, dans la mesure où elle pourrait à la fois être une pépinière de connaissances dans le domaine de l'habitat groupé et un regroupement des acteurs travaillant dans le secteur de l'habitat. 